Dynamic Systems and Applications 30 (2021) No.12, 1779-1791

\title{
EXISTENCE RESULTS FOR FRACTIONAL DIFFERENTIAL INCLUSIONS
}

\author{
AMOURIA HAMMOU AND SAMIRA HAMANI \\ Laboratoire des Mathématiques Appliqués et Pures, Université de Mostaganem, \\ B.P. 227, 27000, Mostaganem, Algeria.
}

\begin{abstract}
In this paper, we investigate the existence of a boundary value problem for CaputoHadamard fractional differential inclusions. Both cases of convex and nonconvex valued right hand side are considered.
\end{abstract}

AMS (MOS) Subject Classification. 26A33, 34A37.

Key Words and Phrases. Fractional differential inclusions, Caputo-Hadamard fractional derivative, Fixed point, Convex, Nonconvex. .

\section{INTRODUCTION}

This paper is concerned with the existence of solutions of boundary value problems (BVP for short) for a fractional differential inclusion,

$$
\begin{gathered}
{ }_{H}^{c} D^{r} y(t) \in F(t, y(t)), \text { for a.e. } t \in J=[1, T], \quad 0<r \leq 1, \\
a y(1)+b y(T)=c,
\end{gathered}
$$

where $T>1,{ }_{H}^{c} D^{r}$ is the Caputo-Hadamard fractional derivative of order $0<r \leq 1$, $F:[1, T] \times \mathbb{R} \rightarrow P(\mathbb{R})$ is a multivalued map, $P(\mathbb{R})$ is the family of all nonempty subsets of $\mathbb{R}, a, b$ and $c$ are real constants such that $a+b \neq 0$.

Differential equations of fractional order have recently proved to be valuable tool$\mathrm{s}$ in the modeling of many phenomena in various fields of science and engineering. Indeed, we can find numerous applications in viscoelasticity, electrochemistry, control, porous media, electromagnetic, etc. (see [15, 24, 32, 33, 36]). However, the literature on Hadamard-type fractional differential equations has not undergone as much development; see [4]. Hadamard's fractional derivative [22] of 1892 differs from the aforementioned derivatives in the sense that the kernel of the integral in the definition of the Hadamard derivative contains a logarithmic function of arbitrary exponent. Detailed descriptions of the Hadamard fractional derivative and integral can

Received August 22, 2021

$\$ 15.00$ (C) Dynamic Publishers, Inc. www.dynamicpublishers.org.
ISSN 1056-2176(Print); ISSN 2693-5295 (online) https://doi.org/10.46719/dsa202130.12.03 
be found in $[9,10,11]$. Recently, Hadamard fractional calculus is getting attention important to the theory of fractional calculus [28]. The works in $[4,9,10,11,27,30]$ are major developments in the fundamental theory of Hadamard fractional calculus. A Caputo-type modification of the Hadamard fractional derivative, which is called the Caputo-Hadamard fractional derivative, was given in [25], and its fundamental theorems were proved in $[1,20]$.

This paper is organized as follows. In Section 2 we introduce some preliminary results needed in the following sections. In Section 3 we present an existence result for the problem (1.1)-(1.2), when the right hand side is convex valued using the nonlinear alternative of Leray-Schauder type. In Section 4,we give a result for nonconvex valued right hand side where is based on a fixed point theorem due to Covitz and Nadler [13]. An example is presented in the last section.

\section{PRELIMINARIES}

In this section, we introduce notations, definitions, and preliminary facts that are used in the remainder of this paper.

Let $[a, b]$ be a compact interval, $C([a, b], \mathbb{R})$ be the Banach space of all continuous functions from $[a, b]$ into $\mathbb{R}$ with the norm

$$
\|y\|_{\infty}=\sup \{|y(t)|: a \leq t \leq b\}
$$

and we denote by $L^{1}([a, b], \mathbb{R})$ the Banach space of functions $y:[a, b] \rightarrow \mathbb{R}$ that are Lebesgue integrable with norm

$$
\|y\|_{L^{1}}={ }_{a}^{b}|y(t)| d t
$$

$A C([a, b], R)$ is the space of functions $y:[a, b] \rightarrow \mathbb{R}$, which are absolutely continuous. Let $(X,\|\cdot\|)$ be a Banach space. Let $P_{c l}(X)=\{Y \in P(X): Y$ is closed $\}, P_{b}(X)=$ $\{Y \in P(X): Y$ is bounded $\}, P_{c p}(X)=\{Y \in P(X): Y$ is compact $\}$ and $P_{c p, c}(X)=$ $\{Y \in P(X): Y$ is compact and convex $\}$. A multivalued map $G: X \rightarrow P(X)$ is convex (closed) valued if $G(X)$ is convex (closed) for all $x \in X$. G is bounded on bounded sets if $G(B)=\cup_{x \in B} G(x)$ is bounded in $X$ for all $B \in P_{b}(X)$ (i.e. $\sup _{x \in B}\{\sup \{|y|$ : $y \in G(x)\}\}<\infty)$.

$G$ is called upper semi-continuous (u.s.c.) on $X$ if for each $x_{0} \in X$, the set $G\left(x_{0}\right)$ is a nonempty closed subset of $X$, and if for each open set $N$ of $X$ containing $G\left(x_{0}\right)$, there exists an open neighborhood $N_{0}$ of $x_{0}$ such that $G\left(N_{0}\right) \subseteq N$. G is said to be completely continuous if $G(B)$ is relatively compact for every $B \in P_{b}(X)$.

If the multivalued map $G$ is completely continuous with nonempty compact values, then $G$ is u.s.c. if and only if $G$ has a closed graph (i.e. $x_{n} \rightarrow x_{*}, y_{n} \rightarrow y_{*}, y_{n} \in$ $G\left(x_{n}\right)$ imply $\left.y_{*} \in G\left(x_{*}\right)\right)$. $G$ has a fixed point if there is $x \in X$ such that $x \in G(x)$. 
The fixed point set of the multivalued operator $G$ will be denote by FixG. A multivalued map $G: J \rightarrow P_{c l}(\mathbb{R})$ is said to be measurable if for every $y \in \mathbb{R}$, the function

$$
t \rightarrow d(y, G(t))=\inf \{|y-z|: z \in G(t)\}
$$

is measurable.

Let $A$ be a subset of $[0, T] \times \mathbb{R} . \quad A$ is $l \otimes \beta$ measurable if $A$ belongs to the $\sigma$-algebra generated by all sets of the form $J \times D$ where $J$ is Lebesgue measurable in $[0, T]$ and $D$ is Borel measurable in $\mathbb{R}$. A subset $A$ of $L^{1}([0, T], \mathbb{R})$ is decomposable if for all $u, v \in A$ and $J \subset[0, T]$ measurable, $u \chi_{J}+v \chi_{[a, b]-J} \in A$, where $\chi$ stands for the characteristic function.

Definition 2.1. A function $F:[a, b] \times \mathbb{R} \rightarrow P(\mathbb{R})$ is said to be Caratheódory if

(1) $t \rightarrow F(t, u)$ is measurable for each $u \in \mathbb{R}$;

(2) $u \rightarrow F(t, u)$ is upper semicontinuous for almost all $t \in[a, b]$.

For each $y \in C([a, b], \mathbb{R})$, define the set of selections of $F$ by

$$
S_{F, y}=\left\{v \in L^{1}([a, b], \mathbb{R}): v(t) \in F(t, y(t)) \text { a.e. } t \in[a, b]\right\} .
$$

Let $(X, d)$ be a metric space induced from the normed space $(X,|\cdot|)$. Consider $H_{d}: P(X) \times P(X) \rightarrow \mathbb{R}_{+} \cup\{\infty\}$ given by

$$
H_{d}(A, B)=\max \left\{\sup _{a \in A} d(a, B), \sup _{b \in B} d(A, b)\right\},
$$

where $d(A, b)=\inf _{a \in A} d(a, b), d(a, B)=\inf _{b \in B} d(a, b)$. Then $\left(P_{b, c l}(X), H_{d}\right)$ is a metric space and $\left(P_{c l}(X), H_{d}\right)$ is a generalized metric space (see [29]).

Definition 2.2. A multivalued operator $N: X \rightarrow P_{c l}(X)$ is called

(1) $\gamma$-Lipschitz if and only if there exists $\gamma>0$ such that

$$
H_{d}(N(x), N(y)) \leq \gamma d(x, y), \quad \text { for each } x, y \in X,
$$

(2) a contraction if and only if it is $\gamma$-Lipschitz with $\gamma<1$.

The following lemma will be used in the sequel.

Lemma 2.3. (Covitz-Nadler [13]) Let $(X, d)$ be a complete metric space. If $N: X \rightarrow$ $P_{c l}(X)$ is a contraction, then Fix $N \neq \emptyset$.

Theorem 2.4. (Arzela-Ascoli theorem)[?] Let $A$ be a subset of $C(J ; E) ; A$ is relatively compact in $C(J ; E)$ if and only if the following conditions are met:

(a) The set $A$ is bounded ie:

$\exists k>0:\|f(x)\| \leq k, \forall x \in J$ and $\forall f \in A$.

(b) Set $A$ is equicontinuous ie:

$\forall \epsilon>0, \exists \delta>0:\left|t_{1}-t_{2}\right|<\delta \Rightarrow\left\|f\left(t_{1}\right)-f\left(t_{2}\right)\right\| \leq \epsilon$ for all $t_{1}, t_{2} \in J$ and all $f \in$ $A$. 
(c) For all $x \in J:$ set $\{f(x), f \in A\} \subset E$ is relatively compact.

Theorem 2.5. (Mazur) Let $\left\{x_{n}\right\}$ be a weakly convergent sequence to $x$ in a Banach space $E$. Then, there is a sequence of convex combination of elements of $\left\{x_{n}\right\}$ which converges strongly to $x$.

Definition 2.6. ([28]) The Hadamard fractional integral of order $\alpha>0$ for a function $h:[a, b] \rightarrow \mathbb{R}$, where $a, b \geq 0$, is defined by

$$
I_{a}^{\alpha} h(t)=1 \Gamma(\alpha)_{a}^{t}(\log t s)^{\alpha-1} h(s) s d s
$$

provided the integral exists.

Definition 2.7. ([25]). Let $A C_{\delta}^{n}[a, b]=\left\{g:[a, b] \rightarrow \mathbb{C}, \delta^{n-1} g \in A C[a, b]\right\}$ where $\delta=t d d t, 0<a<b<\infty$ and let $\alpha \in \mathbb{C}$, such that $\operatorname{Re}(\alpha) \geq 0$. For a function $g \in A C_{\delta}^{n}[a, b]$ the Caputo-Hadamard derivative of fractional order $\alpha$ is defined as follows

(i): If $\alpha \notin \mathbb{N}$, and $n-1<\alpha<n$ such that $n=[\operatorname{Re}(\alpha)]+1$, then

$$
\left({ }^{C H} D_{a}^{\alpha} g\right)(t)=1 \Gamma(n-\alpha)(t d d t)_{a}^{n t}(\log t s)^{n-\alpha-1} \delta^{n} g(s) \frac{d s}{s},
$$

(ii): If $\alpha=n \in \mathbb{N}$, then $\left({ }^{C H} D_{a}^{\alpha} g\right)(t)=\delta^{n} g(t)$,

where in both cases, $[\operatorname{Re}(\alpha)]$ denotes the integer part of the real number $\operatorname{Re}(\alpha)$ and $\log (\cdot)=\log _{e}(\cdot)$.

Lemma 2.8. ([25]) Let $y \in A C_{\delta}^{n}[a, b]$ or $C_{\delta}^{n}[a, b]$ and $\alpha \in \mathbb{C}$. Then

$$
I_{a}^{\alpha}\left({ }^{C H} D_{a}^{\alpha} y\right)(t)=y(t)-\sum_{k=0}^{n-1} \frac{\delta^{k} y(a)}{k !}\left(\log \frac{t}{a}\right)^{k} .
$$

Let us now recall the nonlinear alternative of Leray-Schauder.

Theorem 2.9. [21] Let $X$ be a Banach space and $C$ a nonempty convex subset of $X$. Let $U$ a nonempty open subset of $C$ with $0 \in U$ and $T: \bar{U} \rightarrow C$ continuous and compact operator.

Then either

(a) Thas fixed points. Or

(b) There exist $u \in \partial U$ and $\lambda \in[0,1]$ with $x=\lambda T(x)$.

\section{MAIN RESULTS}

3.1. The convex case. Let us start by defining what we mean by a solution of the problem (1.1)-(1.2).

Definition 3.1. A function $y \in A C_{\delta}^{1}(J, \mathbb{R})$ is said to be a solution of (1.1)-(1.2), if there exists a function $v \in C(J, \mathbb{R})$ with $v(t) \in F(t, y(t))$, for a.e.t $\in J$ such that ${ }_{H}^{c} D^{r} y(t)=v(t)$, and the function $y$ satisfies condition (1.2). 
To prove the existence of a solution to (1.1)-(1.2), we need the following auxiliary lemma

Lemma 3.2. Let $h:[1,+\infty) \rightarrow \mathbb{R}$ be a continuous function. A function $y$ is a solution of the fractional integral equation

$$
y(t)=1 \Gamma(r)_{1}^{t}(\log t s)^{r-1} h(s) d s s-b \Gamma(r)(a+b)_{1}^{T}(\log T s)^{r-1} h(s) d s s+c(a+b)
$$

if and only if $y$ is a solution of the fractional boundary value problem,

$$
\begin{gathered}
{ }_{H}^{c} D^{r} y(t)=h(t), \quad 0<r \leq 1, \\
a y(1)+b y(T)=c,
\end{gathered}
$$

Proof: Assume $y$ satisfies (3.2). Then Lemma (2.8) implies that

$$
y(t)={ }_{H} I^{r} h(t)+y(1) .
$$

The boundary condition (3.3)implies that

$$
\begin{gathered}
a y(1)+b y(T)={ }_{H}^{c} I^{r} h(t)+(a+b) y(1)=c . \\
y(1)=c a+b-b_{H} I^{r} h(t) a+b .
\end{gathered}
$$

Finally, we obtain the solution (3.1)

$$
y(t)={ }_{H} I^{r} h(t)-b a+b_{H} I^{r} h(t)+c a+b .
$$

Conversely it is clear that if $y$ satisfies equation (3.1), then equations (3.2)-(3.3) hold.

Theorem 3.3. Assume the following hypotheses hold:

(H1) $\quad F: J \times \mathbb{R} \longrightarrow P_{c p, c}(\mathbb{R})$ is a Carathéodory multi-valued map;

(H2) There exist $p \in C\left(J, \mathbb{R}^{+}\right)$and $\psi:[0, \infty) \rightarrow(0, \infty)$ continuous and nondecreasing such that

$$
\|F(t, u)\|_{P} \leq p(t) \psi(|u|) \text { for } t \in J \text { and each } u \in \mathbb{R}
$$

(H3) There exists $l \in L^{1}(J, \mathbb{R})$, with $I^{r} l<\infty$ such that

$$
H_{d}(F(t, u), F(t, \bar{u})) \leq l(t)|u-\bar{u}| \text { for every } u, \bar{u} \in \mathbb{R}
$$

and

$$
d(0, F(t, 0)) \leq l(t), \text { a.e. } t \in J .
$$

(H4) There exists a number $M>0$ such that

$$
M[1+|b||a+b|] \psi(M)_{H} I^{r} p(T)+|c||a+b|>1 .
$$

Then the BVP (1.1)-(1.2) has at least one solution on J. 
Proof Transform the problem (1.1)-(1.2) into a fixed point problem. Consider the multivalued operator

$$
N(y(t))=\left\{h \in C(J, \mathbb{R}): \begin{array}{rl}
h(t) & =1 \Gamma(r)_{1}^{t}(\log t s)^{r-1} v(s) d s s \\
& -1 a+b\left[b \Gamma(r)_{1}^{T}(\log T s)^{r-1} v(s) d s s-c\right], v \in S_{F, y} .
\end{array}\right\}
$$

Remark 3.4. Clearly, from Lemma (3.2), the fixed points of $N$ are solutions to $(1.1)-(1.2)$.

We shall show that $N$ satisfies the assumptions of the nonlinear alternative of Leray-Schauder type. The proof will be given in several steps.

Step 1: $N(y)$ is convex for each $y \in C(J, \mathbb{R})$.

Indeed, if $h_{1}, h_{2}$ belong to $N(y)$, then there exist $v_{1}, v_{2} \in S_{F, y}$ such that for each $t \in J$ we have

$$
\begin{aligned}
h_{i}(t) & =1 \Gamma(r)_{1}^{t}(\log t s)^{r-1} v_{i}(s) d s s \\
& -1 a+b\left[b \Gamma(r)_{1}^{T}(\log T s)^{r-1} v_{i}(s) d s s-c\right], \quad i=1,2 .
\end{aligned}
$$

Let $0 \leq d \leq 1$. Then, for each $t \in J$, we have

$$
\begin{aligned}
\left(d h_{1}+(1-d) h_{2}\right)(t) & =1 \Gamma(r)_{1}^{t}\left(\log \frac{t}{s}\right)^{r-1}\left[d v_{1}(s)+(1-d) v_{2}(s)\right] \frac{d s}{s} \\
& -1 a+b\left[b \Gamma(r)_{1}^{T}\left(\log \frac{T}{s}\right)^{r-1}\left[d v_{1}(s)+(1-d) v_{2}(s)\right] \frac{d s}{s}-c\right] .
\end{aligned}
$$

Since $S_{F, y}$ is convex (because $F$ has convex values), we have

$$
d h_{1}+(1-d) h_{2} \in N(y) .
$$

Step 2: $N$ maps bounded sets into bounded sets in $C(J, \mathbb{R})$.

Let $B_{\eta^{*}}=\left\{y \in C(J, \mathbb{R}):\|y\|_{\infty} \leq \eta^{*}\right\}$ be bounded set in $C(J, \mathbb{R})$ and $y \in B_{\eta^{*}}$. Then for each $h \in N(y)$, there exists $v \in S_{F, y}$ such that

$$
\begin{aligned}
h(t) & =1 \Gamma(r)_{1}^{t}(\log t s)^{r-1} v(s) d s s \\
& -1 a+b\left[b \Gamma(r)_{1}^{T}(\log T s)^{r-1} v(s) d s s-c\right] .
\end{aligned}
$$

By (H2) we have for each $t \in J$,

$$
\begin{aligned}
|h(t)| & \leq 1 \Gamma(r)_{1}^{t}(\log t s)^{r-1}|v(s)| d s s \\
& +|b| \Gamma(r)|a+b|_{1}^{T}(\log T s)^{r-1}|v(s)| d s s+|c||a+b| \\
& \leq 1 \Gamma(r)_{1}^{t}(\log t s)^{r-1} \mid p(s) \psi(|y(s)|) d s s \\
& +|b| \Gamma(r)|a+b|_{1}^{T}(\log t s)^{r-1} p(s) \psi(|y(s)|) d s s+|c||a+b| \\
& \leq \psi\left(\eta^{*}\right)_{H} I^{r}(p)(T)+|b| \psi\left(\eta^{*}\right)_{H} I^{r}(p)(T)|a+b|+|c||a+b| .
\end{aligned}
$$

Thus 


$$
\|h\|_{\infty} \leq\left(1+|b||a+b| \eta^{*}\right) \psi\left(\eta^{*}\right)_{H} I^{r} p(T)+|c||a+b|:=l
$$

Step 3: $N$ maps bounded sets into equicontinuous sets of $C(J, \mathbb{R})$.

Let $t_{1}, t_{2} \in J, \quad t_{1}<t_{2}, B_{\eta^{*}}$ be a bounded set of $C\left(J, \mathbb{R}^{+}\right)$as in Step 2, let $y \in B_{\eta^{*}}$ and $h \in N(y)$, then

$$
\begin{aligned}
\left|h\left(t_{2}\right)-h\left(t_{1}\right)\right|= & \mid 1 \Gamma(r)_{1}^{t_{1}}\left[\left(\log t_{1} s\right)^{r-1}-(\log t s)^{r-1}\right] v(s) d s s \\
& +1 \Gamma(r)_{t_{1}}^{t_{2}}(\log t s)^{r-1}|v(s) d s s| \\
\leq & \|p\|_{\infty} \psi\left(\eta^{*}\right) \Gamma(r)_{1}^{t_{1}}\left[\left(\log t_{1} s\right)^{r-1}-\left(\log t_{2} s\right)^{r-1} \mid\right] d s s \\
& +\mid p \|_{\infty} \psi\left(\eta^{*}\right) \Gamma(r)_{t_{1}}^{t_{2}}\left(\log t_{2} s\right)^{r-1} d s s \\
\leq & \|p\|_{\infty} \psi\left(\eta^{*}\right) \Gamma(r+1)\left[\left(\log \left(t_{2}\right)-\log \left(t_{1}\right)\right)^{r}+\log \left(t_{1}\right)^{r}-\log \left(t_{2}\right)^{r}\right]+\|p\|_{\infty} \psi\left(\eta^{*}\right) \Gamma(r+1) \\
\leq & \|p\|_{\infty} \psi\left(\eta^{*}\right) \Gamma(r+1)\left(\log \left(t_{2}\right)-\log \left(t_{1}\right)\right)^{r}+\|p\|_{\infty} \psi\left(\eta^{*}\right) \Gamma(r+1)\left(\log \left(t_{1}\right)^{r}-\log \left(t_{2}\right)^{r}\right)
\end{aligned}
$$

As $t_{1} \longrightarrow t_{2}$, the right-hand side of the above inequality tends to zero. As a consequence of Steps 1 to 3 together with the Arzela-Ascoli theorem, we can conclude that $N: C\left(J, \mathbb{R}^{+}\right) \longrightarrow P(C(J, \mathbb{R}))$ is completely continuous.

Step 4: $N$ has a closed graph.

Let $y_{n} \rightarrow y_{*}, h_{n} \in N\left(y_{n}\right)$ and $h_{n} \rightarrow h_{*}$. We need to show that $h_{*} \in N\left(y_{*}\right)$. $h_{n} \in N\left(y_{n}\right)$ means that there exists $v_{n} \in S_{F, y_{n}}$ such that, for each $t \in J$,

$$
\begin{aligned}
h_{n}(t) & =1 \Gamma(r)_{1}^{t}(\log t s)^{r-1} v_{n}(s) d s s \\
& -1 a+b\left[b \Gamma(r)_{1}^{T}(\log t s)^{r-1} v_{n}(s) \frac{d s}{s}-c\right] .
\end{aligned}
$$

We must show that there exists $v_{*} \in S_{F, y_{*}}$ such that, for each $t \in J$,

$$
\begin{aligned}
h_{*}(t) & =1 \Gamma(r)_{1}^{t}(\log t s)^{r-1} v_{*}(s) d s s \\
& -1 a+b\left[b \Gamma(r)_{1}^{T}(\log t s)^{r-1} v_{*}(s) d s s-c\right] .
\end{aligned}
$$

Since $F(t, \cdot)$ is upper semicontinuous, then for every $\varepsilon>0$, there exist $n_{0}(\epsilon) \geq 0$ such that for every $n \geq n_{0}$, we have

$$
v_{n}(t) \in F\left(t, y_{n}(t)\right) \subset F\left(t, y_{*}(t)\right)+\varepsilon B(0,1) \text {, a.e. } t \in J .
$$

Since $F(\cdot, \cdot)$ has compact values, then there exists a subsequence $v_{n_{m}}(\cdot)$ such that

$$
v_{n_{m}}(\cdot) \rightarrow v_{*}(\cdot) \text { as } m \rightarrow \infty
$$

and

$$
v_{*}(t) \in F\left(t, y_{*}(t)\right) \text {, a.e. } t \in J .
$$

For every $w \in F\left(t, y_{*}(t)\right)$, we have

$$
\left|v_{n_{m}}(t)-v_{*}(t)\right| \leq\left|v_{n_{m}}(t)-w\right|+\left|w-v_{*}(t)\right| .
$$


Then

$$
\left|v_{n_{m}}(t)-v_{*}(t)\right| \leq d\left(v_{n_{m}}(t), F\left(t, y_{*}(t)\right) .\right.
$$

By an analogous relation, obtained by interchanging the roles of $v_{n_{m}}$ and $v_{*}$, it follows that

$$
\left|v_{n_{m}}(t)-v_{*}(t)\right| \leq H_{d}\left(F\left(t, y_{n}(t)\right), F\left(t, y_{*}(t)\right)\right) \leq l(t)\left\|y_{n}-y_{*}\right\|_{\infty} .
$$

Then

$$
\begin{aligned}
\left|h_{n}(t)-h_{*}(t)\right| & \leq 1 \Gamma(r)_{1}^{t}(\log t s)^{r-1}\left|v_{n_{m}}(s)-v_{*}(s)\right| d s s \\
& +|b||a+b| 1 \Gamma(r)_{1}^{T}(\log T s)^{r-1}\left|v_{n_{m}}(s)-v_{*}(s)\right| d s s \\
& \leq 1 \Gamma(r)_{1}^{t}(\log t s)^{r-1} l(s) \frac{d s}{s}\left\|y_{n_{m}}-y_{*}\right\|_{\infty} \\
& +|b||a+b| 1 \Gamma(r)_{1}^{T}(\log T s)^{r-1} l(s) d s s\left\|y_{n_{m}}-y_{*}\right\|_{\infty} .
\end{aligned}
$$

Hence

$$
\begin{aligned}
\left\|h_{n_{m}}-h_{*}\right\|_{\infty} & \leq 1 \Gamma(r)_{1}^{t}(\log t s)^{r-1} l(s) d s s\left\|y_{n_{m}}-y_{*}\right\|_{\infty} \\
& +|b||a+b| 1 \Gamma(r)_{1}^{T}(\log T s)^{r-1} l(s) d s s\left\|y_{n_{m}}-y_{*}\right\|_{\infty} \rightarrow 0 \text { as } m \rightarrow \infty .
\end{aligned}
$$

\section{Step 5: A priori bounds on solutions.}

Let $y$ be a possible solution of the problem (1.1)-(1.2). Then, there exists $v \in S_{F, y}$ such that, for each $t \in J$,

$$
\begin{aligned}
|y(t)| & \leq 1 \Gamma(r)_{1}^{t}(\log t s)^{r-1}|v(s)| d s s \\
& +|b| \Gamma(r)|a+b|_{1}^{T}(\log T s)^{r-1}|v(s)| d s s+|c||a+b| \\
& \leq 1 \Gamma(r)_{1}^{t}(\log t s)^{r-1} p(s) \psi(|y(s)|) d s s \\
& +|b| \Gamma(r)|a+b|_{1}^{T}(\log T s)^{r-1} p(s) \psi(|y(s)|) d s s+|c||a+b| \\
& \leq \psi\left(\|y\|_{\infty}\right) \Gamma(r)_{1}^{t}(\log t s)^{r-1} p(s) d s s \\
& +|b| \psi\left(\|y\|_{\infty}\right) \Gamma(r)|a+b|_{1}^{T}(\log T s)^{r-1} p(s) d s s+|c||a+b| \\
& \leq \psi\left(\|y\|_{\infty}\right)\left({ }_{H} I^{r} p\right)(T)+|b| \psi\left(\|y\|_{\infty}\right)\left({ }_{H} I^{r} p\right)(T)|a+b|+|c||a+b| .
\end{aligned}
$$

Thus

$$
\begin{aligned}
y(t) & =1 \Gamma(r)_{1}^{t}(\log t s)^{r-1} v(s) d s s \\
& -1 a+b\left[b \Gamma(r)_{1}^{T}(\log T s)^{r-1} v(s) d s s-c\right] .
\end{aligned}
$$

This implies by (H2) that, for each $t \in J$, we have

$$
\|y\|_{\infty}[1+|b||a+b|] \psi\left(\|y\|_{\infty}\right)_{H} I^{r} p(T)+|c||a+b|<1 .
$$

Then by condition (3.8), there exists $M$ such that $\|y\|_{\infty} \neq M$. 
Let

$$
U=\left\{y \in C(J, \mathbb{R}):\|y\|_{\infty}<M\right\} .
$$

The operator $N: \bar{U} \rightarrow P(C(J, \mathbb{R}))$ is upper semicontinuous and completely continuous. From the choice of $U$, there is no $y \in \partial U$ such that $y \in \lambda N(y)$ for some $\lambda \in(0,1)$. As a consequence of the nonlinear alternative of Leray-Schauder type, we deduce that $N$ has a fixed point $y$ in $\bar{U}$ which is a solution of the problem (1.1)-(1.2). This completes the proof.

3.2. The Nonconvex case. We present now a result for the problem (1.1)-(1.2) with a nonconvex valued right hand side. Our considerations are based on the fixed point theorem for contraction multivalued maps given by Covitz and Nadler [13].

Theorem 3.5. Assume (H3) and the following hypothesis holds:

(H5) $F: J \times \mathbb{R} \longrightarrow P_{c p}(\mathbb{R})$ has the property that $F(\cdot, u): J \rightarrow P_{c p}(\mathbb{R})$ is measurable for each $u \in \mathbb{R}$;

If

$$
\left\|I^{r} l\right\|_{\infty}(1+|b||a+b|)<1
$$

then the BVP (1.1)-(1.2) has at least one solution on $J$.

Remark 3.6. For each $y \in C(J, \mathbb{R})$, the set $S_{F, y}$ is nonempty since by (H5), $F$ has a measurable selection (see [12], Theorem III.6).

Proof. We shall show that $N$ satisfies the assumptions of Lemma 2.3. The proof will be given in two steps.

Step 1: $N(y) \in P_{c l}(C(J, \mathbb{R}))$ for each $y \in C(J, \mathbb{R})$.

Indeed,let $\left(y_{n}\right)_{n \geq 0} \in N(y)$ such that $y_{n} \longrightarrow \tilde{y}$ in $C(J, \mathbb{R})$. Then, $\tilde{y} \in C(J, \mathbb{R})$ and there exists $v_{n} \in S_{F, y}$ such that, for each $t \in J$,

$$
\begin{aligned}
y_{n}(t) & =1 \Gamma(r)_{1}^{t}(\log t s)^{r-1} v_{n}(s) d s s \\
& -1 a+b\left[b \Gamma(r)_{1}^{T}(\log T s)^{r-1} v_{n}(s) d s s-c\right] .
\end{aligned}
$$

Using the fact that $F$ has compact values and from (H3), we may pass to a subsequence if necessary to get that $v_{n}$ converges weakly to $v$ in $L_{w}^{1}(J, \mathbb{R})$. ( the space endowed with the weak topology) An application of Mazur's theorem implies that $v_{n}$ converges strongly to $v$ and hence $v \in S_{F, y}$. Then, for each $t \in J$,

$$
\begin{aligned}
y_{n}(t) \longrightarrow \tilde{y}(t) & =1 \Gamma(r)_{1}^{t}(\log t s)^{r-1} v(s) d s s \\
& -1 a+b\left[b \Gamma(r)_{1}^{T}(\log T s)^{r-1} v(s) d s s-c\right] .
\end{aligned}
$$

So, $\tilde{y} \in N(y)$. 
Step 2: There exists $\gamma<1$ such that

$$
H_{d}(N(y), N(\bar{y})) \leq \gamma\|y-\bar{y}\|_{\infty} \text { for each } y, \bar{y} \in C(J, \mathbb{R})
$$

Let $y, \bar{y} \in C(J, \mathbb{R})$ and $h_{1} \in N(y)$. Then, there exists $v_{1}(t) \in F(t, y(t))$ such that for each $t \in J$

$$
\begin{aligned}
h_{1}(t) & =1 \Gamma(r)_{1}^{t}(\log t s)^{r-1} v_{1}(s) d s s \\
& -1 a+b\left[b \Gamma(r)_{1}^{T}(\log T s)^{r-1} v_{1}(s) d s s-c\right] .
\end{aligned}
$$

From (H3) it follows that

$$
H_{d}(F(t, y(t)), F(t, \bar{y}(t))) \leq l(t)|y(t)-\bar{y}(t)| .
$$

Hence, there exists $w \in F(t, \bar{y}(t))$ such that

$$
\left|v_{1}(t)-w\right| \leq l(t)|y(t)-\bar{y}(t)|, t \in J .
$$

Consider $U: J \rightarrow P(\mathbb{R})$ given by

$$
U(t)=\left\{w \in \mathbb{R}:\left|v_{1}(t)-w\right| \leq l(t)|y(t)-\bar{y}(t)|\right\} .
$$

Since the multivalued operator $V(t)=U(t) \cap F(t, \bar{y}(t))$ is measurable (see Proposition III.4 in [12]), there exists a function $v_{2}(t)$ which is a measurable selection for $V$. So, $v_{2}(t) \in F(t, \bar{y}(t))$, and for each $t \in J$,

$$
\left|v_{1}(t)-v_{2}(t)\right| \leq l(t)|y(t)-\bar{y}(t)| .
$$

Let us define for each $t \in J$

$$
\begin{aligned}
h_{2}(t) & =1 \Gamma(r)_{1}^{t}(\log t s)^{r-1} v_{2}(s) d s s \\
& -1 a+b\left[b \Gamma(r)_{1}^{T}(\log T s)^{r-1} v_{2}(s) d s s-c\right] .
\end{aligned}
$$

Then for $t \in J$

$$
\begin{aligned}
\left|h_{1}(t)-h_{2}(t)\right| & \leq 1 \Gamma(r)_{1}^{t}(\log t s)^{r-1}\left|v_{1}(s)-v_{2}(s)\right| d s s \\
& +|b| \Gamma(r)|a+b|_{1}^{T}(\log T s)^{r-1}\left|v_{1}(s)-v_{2}(s)\right| d s s \\
& \leq 1 \Gamma(r)_{1}^{t}(\log t s)^{r-1}|l(s)||y(s)-\bar{y}(s)| d s s \\
& +|b| \Gamma(r)|a+b|_{1}^{T}(\log T s)^{r-1}|l(s)||y(s)-\bar{y}(s)| \frac{d s}{s} .
\end{aligned}
$$

Thus

$$
\left\|h_{1}-h_{2}\right\|_{\infty} \leq\left[\|\|_{H} I^{r} l \|_{\infty}(1+|b||a+b|)\right]\|y-\bar{y}\|_{\infty} .
$$


By an analogous relation, obtained by interchanging the roles of $y$ and $\bar{y}$, it follows that

$$
H_{d}(N(y), N(\bar{y})) \leq\left[\left\|_{H} I^{r} l\right\|_{\infty}(1+|b||a+b|)\right]\|y-\bar{y}\|_{\infty} .
$$

So by (3.5), $N$ is a contraction and thus, by Lemma $2.3, N$ has a fixed point $y$ which is solution to (1.1)-(1.2). The proof is complete.

3.3. An Example. As an application of the main results, we consider the fractional differential inclusion

$$
\begin{gathered}
{ }_{C}^{H} D^{r} y(t) \in F\left(t, y_{t}\right), \quad \text { for a.e. } t \in J=[1, e], 1<r \leq 2, \\
y(1)+y(e)=0
\end{gathered}
$$

$F:[1, e] \times \mathbb{R} \rightarrow P(\mathbb{R})$ is a multivalued map satisfying

$$
F(t, y)=\left\{v \in \mathbb{R}: f_{1}(t, y) \leq v \leq f_{2}(t, y)\right\}
$$

Where $f_{1}, f_{2}:[1, e] \times \mathbb{R} \mapsto \mathbb{R}$. We assume that for each $t \in[1, e], f_{1}(t, \cdot)$ is lower semi-continuous (i.e., the set $\left\{y \in \mathbb{R}: f_{1}(t, y)>\mu_{1}\right\}$ is open for each $\mu_{1} \in \mathbb{R}$ ), and assume that for each $t \in[1, e], f_{2}(t, \cdot)$ is upper semi-continuous (i.e., the set the set $\left\{y \in \mathbb{R}: f_{2}(t, y)<\mu_{2}\right\}$ is open for each $\left.\mu_{2} \in \mathbb{R}\right)$. Assume that there is a function $p \in C\left(J, \mathbb{R}^{+}\right)$and $\psi:[0, \infty) \rightarrow(0, \infty)$ continuous and nondecreasing such that

$$
\begin{aligned}
\|F(t, y)\|_{P} & =\sup \{|v|: v(t) \in F(t, y)\} \\
& =\max \left(\left|f_{1}(t, y)\right|,\left|f_{2}(t, y)\right|\right) \leq p(t) \psi(|y|), \quad \text { for each } t \in[1, e], y \in \mathbb{R} .
\end{aligned}
$$

Where

$$
a=b=1, c=0, T=e
$$

and there exists a number $M>0$ such that

$$
M[32] \psi(M)_{H} I^{r} p(e)>1 .
$$

Since all the conditions of Theorem 3.3 are satisfied, problem (3.6)-(3.7) has at least one solution $y$ on $[1, e]$.

\section{REFERENCES}

[1] Y. Adjabi, F. Jarad, D. Baleanu and T. Abdeljawad, On Cauchy problems with Caputo Hadamard fractional derivatives, J. Comput. Anal. Appl. 21 (4) (2016), 661-681.

[2] R. P Agarwal, M. Benchohra and S. Hamani, Boundary value problems for fractional differential equations, Adv. Stud. Contemp. Math. 16 (2) (2008), 181-196.

[3] B. Ahmad, R. A. Khan and S. Sivasundaram, Generalized quasilinearization method for a first order differential equation with integral boundary condition, J. Cont., Disc. Impulsive Sys. Ser. A Math. Anal. 12 (2) (2005), 289-296.

[4] B. Ahmad and S. K. Ntouyas, Initial value problems for hybrid Hadamard fractional equations, Electron. J. Differential Equations 2014 (2014), No. 161, 8 pp.

[5] M. Benchohra and S. Hamani, Boundary value problems for differential inclusions with fractional order,Diss. Math. Dif. Inclusions. Control and Optimization.,28(2008), 147-164. 
[6] M. Benchohra and S. Hamani, Nonlinear boundary value problems for differential inclusions with Caputo fractional derivative, Topol. Methods Nonlinear Anal. 32 (1) (2008), 115-130.

[7] W. Benhamida, S, Hamani and J. Henderson, Boundary Value Problems For CaputoHadamard Fractional Differential Equations, Adv. in the Theo. of Nonl. Analy. and its Appli. 2 (2018) No. 3, 138-145.

[8] M. Benchohra and S. Hamani and S. K. Ntouyas, boundary value problems for differential equations with fractional order, Surv. Math. Appl. 3 (2008), 1-12.

[9] P. L. Butzer, A. A. Kilbas and J. J. Trujillo, Composition of Hadamard-type fractional integration operators and the semigroup property, J. Math. Anal. Appl. 269 (2002), 387-400.

[10] P. L. Butzer, A. A. Kilbas and J. J. Trujillo, Fractional calculus in the Mellin setting and Hadamard-type fractional integrals, J. Math. Anal. Appl. 269 (2002), 1-27.

[11] P. L. Butzer, A. A. Kilbas and J. J. Trujillo, Mellin transform analysis and integration by parts for Hadamard-type fractional integrals, J. Math. Anal. Appl. 270 (2002), 1-15.

[12] C. Castaing and M. Valadier, Convex Analysis and Measurable Multifunctions, Lecture Notes in Mathematics 580, Springer-Verlag, Berlin-Heidelberg-New York, 1977.

[13] H. Covitz and S. B. Nadler, Multivalued contraction mappings in generalized metric spaces, Israel J. Math. 8 (1970), 5-11.

[14] K. Diethelm and N. J. Ford, Analysis of fractional differential equations, J. Math. Anal. Appl. 265 (2002), 229-248.

[15] K. Diethelm and A.D. Freed, On the solution of nonlinear fractional order differential equations used in the modeling of viscoplasticity, in "Scientifice Computing in Chemical Engineering IIComputational Fluid Dynamics, Reaction Engineering and Molecular Properties" (F. Keil, W. Mackens, H. Voss, and J. Werther, Eds), pp 217-224, Springer-Verlag, Heidelberg, 1999.

[16] K. Diethelm and G. Walz, Numerical solution of fractional order differential equations by extrapolation, Numer. Algorithms 16 (1997), 231-253.

[17] A. M. A. El-Sayed, Fractional order diffusion-wave equations, Intern. J. Theo. Physics 35 (1996), 311-322.

[18] A. M. A. El-Sayed, Fractional order evolution equations, J. Fract. Calc. 7 (1995), 89-100.

[19] A. M. A. El-Sayed, Nonlinear functional differential equations of arbitrary orders, Nonlinear Anal. 33 (1998), 181-186.

[20] Y. Y. Gambo, F. Jarad, D. Baleanu and T. Abdeljawad, On Caputo modification of the Hadamard fractional derivatives, Adv. Difference Equ. 2014 (2014), No. 10, 12 pp.

[21] A. Granas and J. Dugundji, Fixed Point Theory, Springer-Verlag, New York, 2003.

[22] J. Hadamard, Essai sur letude des fonctions donnees par leur development de Taylor, J. Math. Pure Appl. 8 (1892), 101-186.

[23] N. Heymans and I. Podlubny, Physical interpretation of initial conditions for fractional differential equations with Riemann-Liouville fractional derivatives. Rheologica Acta 45 (5) (2006), 765-772.

[24] R. Hilfer, Applications of Fractional Calculus in Physics, World Scientific, Singapore, 2000.

[25] F. Jarad, T. Abdeljawad and D. Baleanu, Caputo-type modification of the Hadamard fractional derivatives, Adv. Difference Equ. 2012 (2012), No. 142, 8 pp.

[26] A.A. Kilbas, Hari M. Srivastava, and Juan J. Trujillo, Theory and Applications of Fractional Differential Equations. North-Holland Mathematics Studies, 204. Elsevier Science B.V., Amsterdam, 2006.

[27] A. A. Kilbas, Hadamard-type fractional calculus, J. Korean Math. Soc. 38 (6) (2001), 11911204. 
[28] A. A. Kilbas, H. M. Srivastava and J. J. Trujillo, Theory and Applications of Fractional Differential Equations. North-Holland Mathematics Studies, 204. Elsevier Science B.V., Amsterdam, 2006.

[29] M. Kisielewicz, Differential Inclusions and Optimal Control, Kluwer, Dordrecht, The Netherlands, 1994.

[30] M. Klimek, Sequential fractional differential equations with Hadamard derivative, Commun. Nonlinear Sci. Numer. Simul. 16 (12) (2011), 4689-4697.

[31] A. Lomtatidze and L. Malaguti, On a nonlocal boundary value problems for second order nonlinear singular differential equations, Georg. Math. J. 7 (2000), 133-154.

[32] F. Mainardi, Fractional calculus: Some basic problems in continuum and statistical mechanics, in "Fractals and Fractional Calculus in Continuum Mechanics" (A. Carpinteri and F. Mainardi, Eds), pp. 291-348, Springer-Verlag, Wien, 1997.

[33] F. Metzler, W. Schick, H. G. Kilian and T. F. Nonnenmacher, Relaxation in filled polymers: A fractional calculus approach, J. Chem. Phys. 103 (1995), 7180-7186.

[34] K. S. Miller and B. Ross, An Introduction to the Fractional Calculus and Differential Equations, John Wiley, New York, 1993.

[35] S. M. Momani and S. B. Hadid, Some comparison results for integro-fractional differential inequalities, J. Fract. Calc. 24 (2003), 37-44.

[36] K.B. Oldham and J. Spanier, The Fractional Calculus, Academic Press, New York, London, 1974.

[37] I. Podlubny, Fractional Differential Equations, Academic Press, San Diego, 1999.

[38] I. Podlubny, Geometric and physical interpretation of fractional integration and fractional differentiation, Fract. Calc. Appl. Anal. 5 (2002), 367-386.

[39] S. G. Samko, A. A. Kilbas and O. I. Marichev, Fractional Integrals and Derivatives. Theory and Applications, Gordon and Breach, Yverdon, 1993.

[40] A. M. Samoilenko and N. A. Perestyuk,Impulsive Differential Equations, World Scientific, Singapore, 1995. 